\title{
Tachycardia with wide QRS complex on a cocaine dependent patient: not everything is what it seems
}

Introduction: Ventricular arrhythmias related to cocaine may not respond to antiarrhythmic drugs and may need treatment with radiofrequency ablation.

Case presentation: In this case we describe a 33-year-old man that presented to the emergency room complaining of chest discomfort and slight palpitations predominantly in the precordium, starting for 1 hour ago. The patient reports rare episodes of non tachycardic palpitations in the past, short-lived. He denied syncope or pre- syncope and did not show low output objective signs. After exams, he was diagnosed with sustained ventricular tachycardia confirmed by all used electrocardiographic criteria; the emergency medical team chose to use intravenous amiodarone, which reverted the arrhythmia. The patient was hospitalized, and continued intravenous amiodarone, sedation with benzodiazepines and 24-hour continuous monitoring electrocardiographic (Holter) were conducted. Amiodarone was suspended and was initiated oral diltiazem $80 \mathrm{mg}$ in $8 / 8$ hours. We requested a cardiac nuclear magnetic resonance image that showed normal perfusion and contractility, the absence of delayed enhancement, mild hypertrophy of the basal septum and lack of arrhythmogenic substrate. Electrophysiological study (EPS) was performed.

Conclusion: During the EPS, the ECG at baseline was normal. The programmed electrical stimulation induced atrioventricular nodal reentrant tachycardia (AVNRT) with aberrant conduction. The ablation of the slow pathway was successful, and the patient did not present new tachycardia episodes.

Submitted: 13 June 2016; Accepted: 11 July 2016; Published online: 16 July 2016

Keywords: Cocaine" Ventricular arrhythmias" Atrioventricular nodal reentrant tachycardiaElectrophysiological study= Cardiac ablation

\section{Case Presentation}

Cocaine abuse continues to be a serious problem worldwide [1], and the global number of annual cocaine users in 2010 ranged from 13.3 million to 19.7 million [2]. In the US alone, there were 1.4 million cocaine users in 2011 with as much as $19 \%$ of the US population admitting usage [2].
Unfortunately, in addition to numerous complications in target organs, such as heart, liver, kidney, and central nervous system [3], cocaine is the most frequent cause of drugrelated deaths and emergency room visits in the U.S [4-6]. Since the re- emergence of recreational cocaine use in the late 1980s and the recent appearance of inexpensive freebase
Márcio Galindo Kiuchi',2*, Gustavo Ramalho e Silva', Luis Marcelo Rodrigues $\mathrm{Paz}^{3}$ and Gladyston Luiz Lima Souto' 'Department of Cardiac Surgery and Artificial Cardiac Stimulation, Department of Medicine, Hospital e Clínica São Gonçalo, São Gonçalo, RJ, Brazil

2Electrophysiology Division, Department of Cardiology, Hospital e Clínica São Gonçalo, São Gonçalo, RJ, Brazil ${ }^{3}$ Interventional Cardiology Section, Department of Cardiology, Hospital e Clínica São Gonçalo, São Gonçalo, RJ, Brazil

*Author for correspondence:

Tel.: +552126047744

marciokiuchi@gmail.com 
cocaine, illicit usage of cocaine has been linked to a dramatic rise in both acute and chronic cardio toxicity [7].

While cardio toxic manifestations resemble traditional cardiac conditions symptomatically, the exact mechanism of cocaine-induced cardiac death remains unknown and thus, unpredictable [8-11], leaving the need for a better understanding of both cocaine-related cardiac complications and sudden cardiac death. Cocaine intake is associated with the hypertensive crisis, acute myocardial ischemia/ infarct, ventricular arrhythmias, and congestive heart failure. Some of these effects could be related to its ability to inhibit catecholamine reuptake at the presynaptic nerve endings causing exaggerated sympathetic surge or sodium channel inhibition. Cocaine is known to alter myocardial contractility, decreased the rate of depolarization, diminished action potential amplitude and duration, and conduction. In most subjects with acute cocaine exposure, ventricular arrhythmias are self-limited and resolve with aggressive medical therapy due to its relatively short half-life ( $<10$ hours) [12-14]. However, cocaine can be detected in body fluids for more than a week and may sometimes have prolonged residual effects. Chronic cocaine abuse may lead to myocardial infarction with remodelling and a resultant scar that forms the substrate for ventricular arrhythmias. Although myocardial infarction (MI) is considered the best documented complication of cocaine abuse, little evidence of MI was found during autopsies in which patients died suddenly after cocaine ingestion $[9,11]$. Instead, the cocaine-related cardiac death was most often arrhythmogenic in nature $[9,11]$. Likewise, significant prolongation of the QTc interval among cocaine users has been reported [15-17].

The QTc interval prolongation has been associated with catecholamine-induced polymorphic ventricular tachycardia-related sudden death.

In this report, we describe a 33-year-old man that presented to the emergency room complaining of chest discomfort and slight palpitations predominantly in the precordium, starting for 1 hour ago. The patient reports rare episodes of non tachycardic palpitations in the past, short-lived. He denied syncope or pre-syncope and did not show low output objective signs. He was presenting tachycardia, normal blood pressure, and high heart rate $(\mathrm{HR}=240 \mathrm{bpm})$. The patient claimed to be long time cocaine user and was the last use five days before admission. He made irregular use of naphazoline hydrochloride to treat nasal congestion, denied any chronic illness and had an expressionless family history of the disease. The ECG showed the tachycardia with wide QRS complex, as shown in Figure 1. The ethics committee, comprising Paola Baars Gomes Moises, Luis Marcelo Rodrigues Paz, Humberto Cesar Tinoco and Jonny Shogo Takahashi, approved this case study. Written informed consent was provided by the patient. Multiple algorithms were used by the emergency medical team to confirm that it was a sustained ventricular tachycardia or supraventricular tachycardia with aberrant conduction. The first step was to use the traditional Brugada's algorithm [18] which confirmed that a ventricular tachycardia, because $R$ to $S$ interval $>100 \mathrm{~ms}$ in one precordial lead (V6), as shown in Figure 2A. The next step was to use the other algorithm [19], in which initial $\mathrm{R}$ wave aVR lead was present (Figure $2 \mathrm{~B}$ ), besides broad and notched or slurred $\mathrm{R}$ in $\mathrm{I}$ and V5 and V6, absence of Q wave in I and V5 or V6, and $R$ peak times $\geq 0.06$ s in V5 or V619 (Figure 2C) which was diagnosed ventricular tachycardia. The relationship ventricular Activation-Speed Ventricular $(\mathrm{Vi} / \mathrm{Vt}=2.5$ $\mathrm{mV} / 3 \mathrm{mV}=0.8 \mathrm{mV} \leq 1 \mathrm{mV}$ that suggests ventricular tachycardia), as shown in Figure 2D. Another algorithm [20] suggests ventricular tachycardia showed Notch on the descendant of an early QRS and predominantly negative in the aVR lead (Figure 2E). The R-wave peak time (RWPT) at DII criteria also suggested ventricular tachycardia, because RWPT was $\geq 50 \mathrm{~ms}$ at DII lead (RWPT at DII lead=60 ms, a shown in Figure 2F).

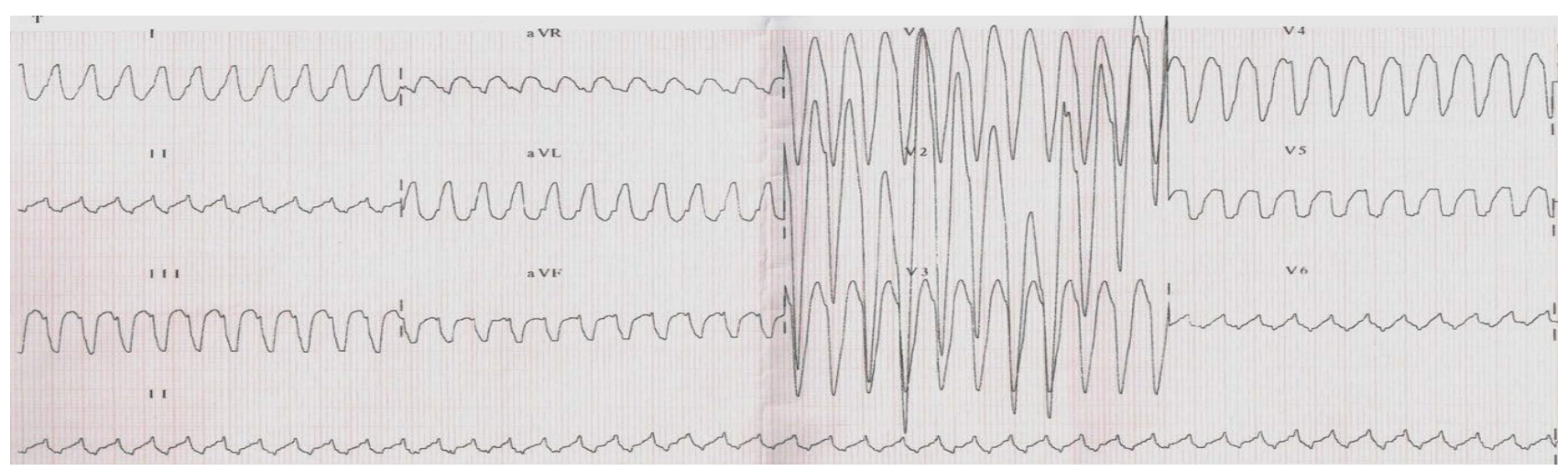

Figure 1: ECG of entrance showing the tachycardia with wide QRS complex. 


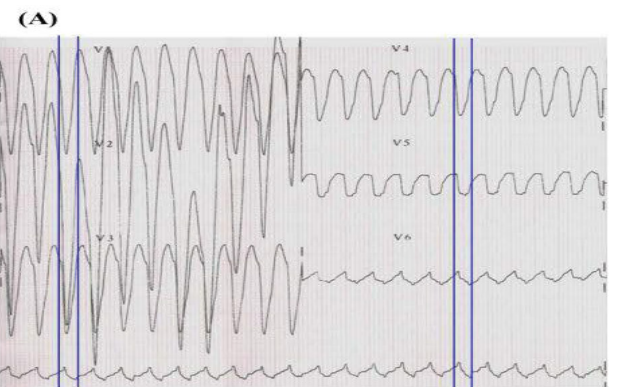

(D)

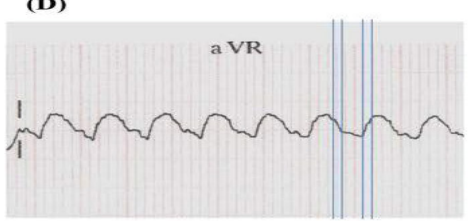

(B)
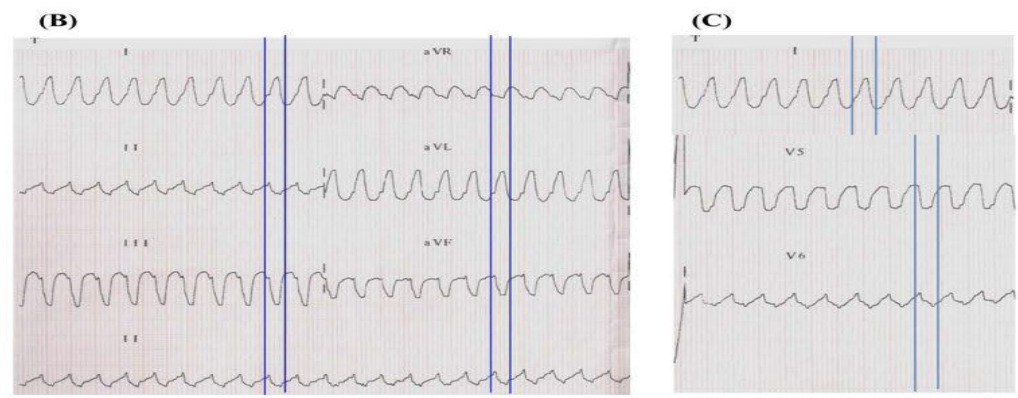

(F)

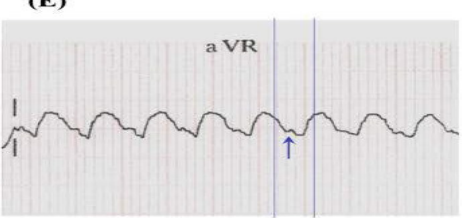

II

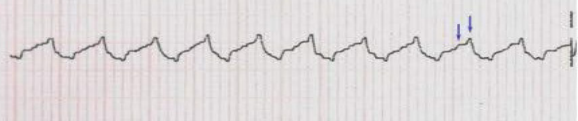

Figure 2: Multiple algorithms were used by the emergency medical team to confirm that it was a sustained ventricular tachycardia or supraventricular tachycardia with aberrant conduction.

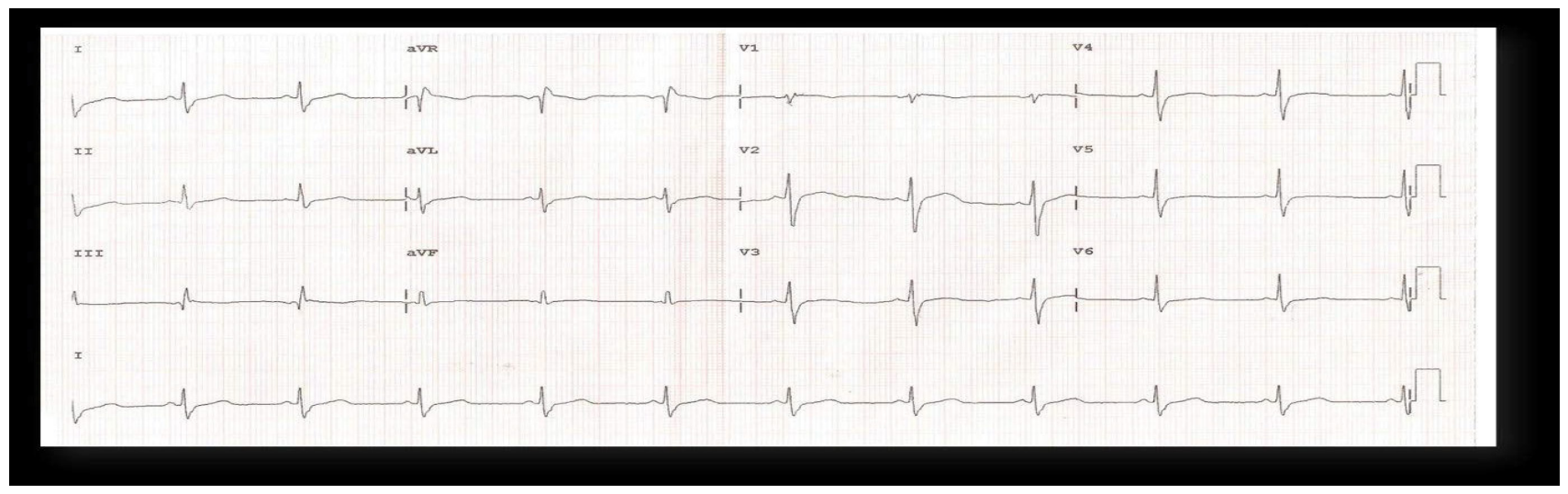

Figure 3: ECG is showing the reversion of the arrhythmia after intravenous amiodarone.

Diagnosed as sustained ventricular tachycardia confirmed by all used electrocardiographic criteria, the emergency medical team chose to use intravenous amiodarone $(300 \mathrm{mg})$, which reverted the arrhythmia (Figure 3). The team of cardiac arrhythmias was called to take up the case. Troponin curve was normal, and the transthoracic echocardiogram showed questionable hypokinesia of the lateral wall of the left ventricle. The patient was hospitalized, and continued intravenous amiodarone, sedation with benzodiazepines and 24 hours continuous monitoring electrocardiographic (Holter) were conducted. The electrocardiogram remained with short PR interval without pre- excitation, Holter monitoring showed no arrhythmia or pre-excitation, and the signalaveraged electrocardiogram was negative. Amiodarone was suspended and was initiated oral diltiazem $80 \mathrm{mg}$ in $8 / 8$ hours. We requested the morphological and perfusion cardiac nuclear magnetic resonance image. This exam revealed normal perfusion and contractility, the absence of delayed enhancement, mild hypertrophy of the basal septum and lack of arrhythmogenic substrate. Our team requested the electrophysiological study (EPS). During the EPS (Figure 4A), the ECG at baseline was normal. The programmed electrical stimulation induced atrioventricular nodal reentrant tachycardia (AVNRT) with aberrant conduction (Figures 4B and 4C). Another programmed electrical stimulation induced atrioventricular nodal reentrant tachycardia (AVNRT) with aberrant conduction and compatible with the clinical arrhythmia shown in the patient's ECG entrance (Figures $4 \mathrm{D}$ and $\mathrm{E})$. Intravenous adenosine was administered, and tachycardia was reversed (Figure 4F). The ablation of the slow pathway was successful, and programmed electrical atrial pacing in the presence of isoproterenol did not induce new arrhythmias (Figure 4G). The programmed ventricular electrical stimulation did not induce any arrhythmia (Figure 4H). The conclusion of the EPS was: AVNRT, ablation of slow pathway, and Ventricular electrical stability. The patient was discharged the next day after the procedure. 

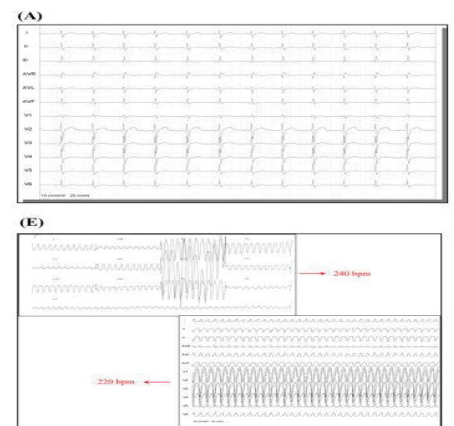
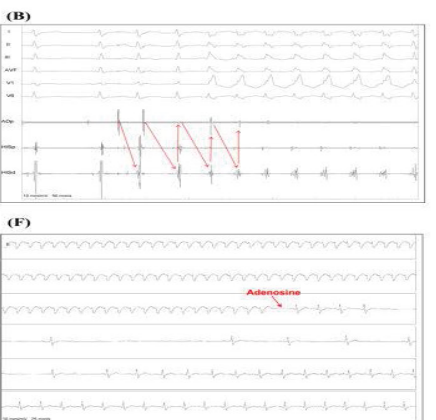

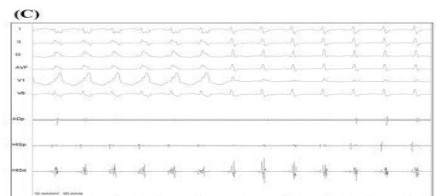

(G)

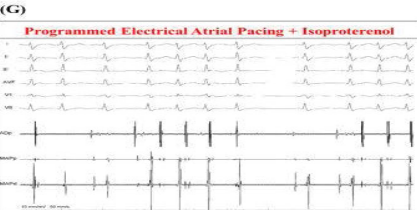

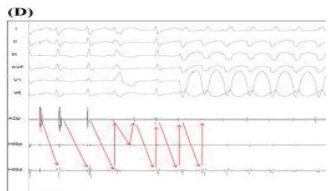

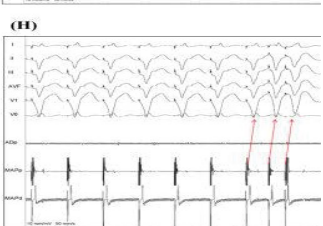

Figure 4: Electrophysiological study and ablation procedure.

Not all tachyarrhythmia seems to be what our eyes see, so we must investigate each arrhythmia judiciously, step by step, and always to the end. In several cases EPS may have a pivotal role to conclude the diagnosis.

\section{References}

1. Calcaterra S, Binswanger IA. National trends in psychostimulant- related deaths: 1999-2009. Subst. Abus. 34, 129-136 (2013).

2. Dargan PI, Wood DM. Recreational drug use in the Asia Pacific region: improvement in our understanding of the problem through the UNODC programmes. J. Med. Toxicol. 8, 295-299 (2012).

3. Valente MJ, Carvalho F, Bastos Md, de Pinho PG, Carvalho M. Contribution of oxidative metabolism to cocaine-induced liver and kidney damage. Curr. Med. Chem. 19, 5601-5606 (2012).

4. National Institute on Drug Abuse. Costs to Society. National Institute on Drug Abuse; Washington, DC: (2002)

5. http://www.samhsa.gov/data/sites/default/files/ DAWN2k11ED/ DAWN2k11ED/DAWN2k11ED.pdf

6. Glauser J, Queen JR. An overview of non-cardiac cocaine toxicity. J. Emerg. Med. 32, 181-186 (2007).

7. Phillips K, Luk A, Soor G, et al. Cocaine cardiotoxicity: a review of the pathophysiology, pathology, and treatment options. Am. J. Cardiovasc. Drugs. 9, 177-196 (2009).

8. Turillazzi E, Bello S, Neri M, et al. Cardiovascular effects of cocaine: cellular, ionic and molecular mechanisms. Curr. Med. Chem. 19, 5664-5676 (2012).

9. Basso C, Marra MP, Thene G. Cocaine and the heart; more than the just coronary disease. Heart. 97, 1995-1996 (2011).

10. Maraj S, Figueredo VM, Lynn Morris D. Cocaine and the heart. Clin. Cardiol. 33, 264-269 (2010).

11. Bauman J, Grawe J, Winecoff A, Hariman R. Cocaine-related

\section{Acknowledgements}

We would like to thank Mr. Sérgio Oliveira and Pacemed for their technical support.

sudden cardiac death: a hypothesis correlating basic science and clinical observations. J. Clin. Pharmacol. 34, 902-911 (1994).

12. Benowitz NL. Clinical pharmacology and toxicology of cocaine. Pharmacol. Toxicol. 72, 3-12 (1993).

13. Fleming JA, Byck R, Barash PG. Pharmacology and therapeutic applications of cocaine. Anesthesiology. 73, 518-531 (1990).

14. Warner EA. Cocaine abuse. Ann. Intern. Med. 119, 226-235 (1993).

15. Levin KH, Copersino ML, Epstein D, Boyd SJ, Gorelick DA Longitudinal ECG changes in cocaine users during extended abstinence. Drug. Alcohol. Depend. 95, 160-163 (2008).

16. Taylor D, Parish D, Thompson L, Cavaliere M. Cocaine induced prolongation of the QT interval. Emerg. Med. J. 21: 252-253 (2004).

17. Gamouras GA, Monir G, Plunkitt K, Gursoy S, Dreifus LS Cocaine abuse: repolarization abnormalities and ventricular arrhythmias. Am. J. Med. Sci. 320, 9-12 (2000).

18. Brugada P, Brugada J, Mont L, Smeets J, Andries EW. A new approach to the differential diagnosis of a regular tachycardia with a wide QRS complex. Circulation. 83, 1649-1659 (1991).

19. Vereckei A, Duray G, Szénási G, Altemose GT, Miller JM. Application of a new algorithm in the differential diagnosis of wide QRS complex tachycardia. Eur. Heart. J. 28, 589-600 (2007).

20. Vereckei A, Duray G, Szénási G, Altemose GT, Miller JM. New algorithm using only lead aVR for differential diagnosis of wide QRS complex tachycardia. Heart. Rhythm. 5, 89-98 (2008). 